\title{
Attenuation of flightless I improves wound healing and enhances angiogenesis in a murine model of type 1 diabetes
}

\author{
Nadira Ruzehaji • Zlatko Kopecki • Elizabeth Melville • \\ Sarah L. Appleby • Claudine S. Bonder • \\ Ruth M. Arkell • Robert Fitridge • Allison J. Cowin
}

Received: 28 August 2013 / Accepted: 18 October 2013 /Published online: 29 November 2013

(C) Springer-Verlag Berlin Heidelberg 2013

\begin{abstract}
Aims/hypothesis Skin lesions and ulcerations are severe complications of diabetes that often result in leg amputations. In this study we investigated the function of the cytoskeletal protein flightless I (FLII) in diabetic wound healing. We hypothesised that overexpression of FLII would have a negative effect on diabetic wound closure and modulation of this protein using specific FLII-neutralising antibodies (FnAb) would enhance cellular proliferation, migration and angiogenesis within the diabetic wound.
\end{abstract}

Electronic supplementary material The online version of this article (doi:10.1007/s00125-013-3107-6) contains peer-reviewed but unedited supplementary material, which is available to authorised users.

N. Ruzehaji $\cdot$ Z. Kopecki $\cdot$ E. Melville $\cdot$ A. J. Cowin

Women's and Children's Health Research Institute,

Adelaide, SA, Australia

N. Ruzehaji • A. J. Cowin

Department of Paediatrics, The University of Adelaide,

Adelaide, SA, Australia

Z. Kopecki $\cdot$ E. Melville $\cdot$ A. J. Cowin $(\bowtie)$

Centre for Regenerative Medicine, Mawson Institute,

University of South Australia, Adelaide, SA 5095, Australia

e-mail: allison.cowin@unisa.edu.au

S. L. Appleby $\cdot$ C. S. Bonder

The Centre for Cancer Biology, Adelaide, SA, Australia

C. S. Bonder

School of Medicine and the Centre for Stem Cell Research,

The University of Adelaide, Adelaide, SA, Australia

R. M. Arkell

Research School of Biology, Australian National University,

Canberra, ACT, Australia

R. Fitridge

Department of Surgery, The University of Adelaide,

Adelaide, SA, Australia
Methods Using a streptozotocin-induced model of diabetes we investigated the effect of altered FLII levels through Flii genetic knockdown, overexpression or treatment with FnAb on wound healing. Diabetic wounds were assessed using histology, immunohistochemistry and biochemical analysis. In vitro and in vivo assays of angiogenesis were used to assess the angiogenic response.

Results FLII levels were elevated in the wounds of both diabetic mice and humans. Reduction in the level of FLII improved healing of murine diabetic wounds and promoted a robust pro-angiogenic response with significantly elevated von Willebrand factor (vWF) and vascular endothelial growth factor (VEGF)-positive endothelial cell infiltration. Diabetic mouse wounds treated intradermally with FnAb showed improved healing and a significantly increased rate of reepithelialisation. FnAb improved the angiogenic response through enhanced formation of capillary tubes and functional neovasculature. Reducing the level of FLII led to increased numbers of mature blood vessels, increased recruitment of smooth muscle actin- $\alpha$-positive cells and improved tight junction formation.

Conclusions/interpretation Reducing the level of FLII in a wound may be a potential therapeutic approach for the treatment of diabetic foot ulcers.

Keywords Angiogenesis · Diabetes · Flightless I · FLII · Flightless neutralising antibodies · Type 1 diabetes · VEGF . Wound healing

$\begin{array}{ll}\text { Abbreviations } \\ \text { FLII } & \text { Flightless I } \\ \text { FnAb } & \text { FLII-neutralising antibodies } \\ \text { H\&E } & \text { Haematoxylin and eosin } \\ \alpha-S M A & \text { Smooth muscle actin- } \alpha \\ \text { STZ } & \text { Streptozotocin } \\ \text { VEGF } & \text { Vascular endothelial growth factor }\end{array}$


vWF von Willebrand factor

WT Wild type

\section{Introduction}

Up to $15 \%$ of people with diabetes can expect to develop a nonhealing ulcer [1], and 3\% will have a lower-limb amputation [2]. Despite numerous approaches the therapeutic results are still very limited. Impaired healing of diabetic wounds is caused by complex factors such as abnormal keratinocyte and fibroblast migration, proliferation, differentiation and apoptosis, and decreased vascularisation [3]. Chronic hyperglycaemia also leads to impaired angiogenesis, which further contributes to the delayed wound healing seen in patients with diabetes $[4,5]$.

The cytoskeletal protein flightless I (FLII) $[6,7]$ regulates the dynamic remodelling of the actin cytoskeleton [8] by severing and bundling actin filaments [9]; it is involved in cellular processes, including migration and proliferation, which are important in wound healing [3]. The defining feature of FLII is its homology with two families of proteins, the gelsolin protein family and the leucine-rich repeat (LRR) protein family [10], providing the capacity to be involved in a variety of signalling pathways [7, 8, 11, 12]. FLII downregulates IL-1/Toll-like receptor (TLR)4 signalling of the TLR pathway, suggesting that FLII is involved in the regulation of the innate immune response [13]. FLII interacts directly with MyD88, an intracellular adaptor protein immediately downstream of most TLRs, and modulates the activity of nuclear factor of $\kappa$ light polypeptide gene enhancer in $\mathrm{B}$ cells $1(\mathrm{NF}-\mathrm{KB})$ [13-15], pro-inflammatory caspases [16] and TNF- $\alpha[10]$. FLII has been identified as an important regulator of wound repair, affecting cell proliferation, motility and matrix deposition [17]. Flii gene heterozygous knockout leads to improved wound healing, whereas its overexpression is associated with impaired healing of wounds [17]. FLIIneutralising antibodies (FnAb) have the capacity to increase cellular proliferation and migration in vitro $[17,18]$ and promote the rate of healing in cutaneous wounds in vivo [19].

Using human samples and genetic mouse models with low $\left(\mathrm{Flii}^{+/-}\right)$, normal $\left(\mathrm{Flii}^{+/+}\right)$, or high $\left(\mathrm{Flii}^{\mathrm{Tg} / \mathrm{Tg}}\right)$ levels of FLII, or $\mathrm{FnAb}$, which binds to extracellular FLII and reduces its local expression, the effect of FLII on diabetic wound healing was investigated. These studies suggest that FLII adversely affects healing in diabetic wounds and that reducing its activity leads to improved wound outcomes and enhanced angiogenesis.

\section{Methods}

Antibodies Mouse monoclonal anti-FLII antibody was obtained from Santa Cruz Biotechnology (Santa Cruz, CA,
USA). Rat anti-mouse CD31 antibody (BD Pharmingen, North Ryde, NSW, Australia), anti-smooth muscle actin- $\alpha$ ( $\alpha$-SMA), rabbit polyclonal anti-vascular endothelial growth factor (VEGF) and rabbit polyclonal anti-von Willebrand factor (vWF) antibodies were obtained from Abcam (Cambridge, UK). Alexa-488- and Alexa-594-tagged secondary antibodies were obtained from Invitrogen (Mulgrave, VIC, Australia) and mouse monoclonal anti-FLIIneutralising antibodies (FnAb) [20] were obtained from MAbSA (Adelaide, SA, Australia).

Human wound and skin collection Prior to recruitment to the study, participants were screened using relevant investigations including blood tests, quantitative microbiology, duplex and advanced wound diagnostics in the form of toe pressure, ankle brachial index and transcutaneous oxygen pressure. Participants with non-diabetic acute wounds (acute trauma wounds such as those seen in the emergency department $[\leq 6$ weeks old]), diabetic wounds (type 1 or type 2 diabetes, diabetic ulcer [ $\geq 6$ weeks old]) and normal unwounded skin (healthy age-matched volunteers) were included in the study. Exclusion criteria for the diabetic wounds included active infection or cellulitis in the area biopsied, drugs that impair wound healing such as steroids and immunosuppressive drugs, ulcer of malignant nature and renal failure/dialysis with GFR $<30 \mathrm{ml} / \mathrm{min}$. As part of the inclusion criteria all patients with diabetic wounds displayed presence of pulses and/or ankle brachial index $>0.7$, and had a diabetic ulcer and toe pressure $>40 \mathrm{mmHg}$. Informed consent was obtained from each patient and the study protocol conformed to the ethical guidelines of the 1975 Declaration of Helsinki as reflected in approval by the Health Service Human Research Ethics Committee and Central Northern Adelaide Health Service Ethics of Human Research Committee. The three study groups were: diabetic ulcer ( $n=6$, mean age 56); acute wound ( $n=6$, mean age 49$)$; and normal skin $(n=6$, mean age 45$)$.

Murine model of type 1 diabetes Flii heterozygous null (Flii ${ }^{\text {Tm1Hdc }}$; Flii ${ }^{+1-}$ ) [9] and transgenic overexpressing (Tg[Flii]2Hdc; Flii ${ }^{T g / T g}$ ) $[17,21]$ mice on a BALB/c background were provided by Ruth M. Arkell, Australian National University, Canberra, ACT, Australia [21]. Wild-type (WT) animals $\left(\mathrm{Flii}^{+/+}\right)$were obtained from the BALB/c colony used for maintenance of the null strain (The University of Adelaide, Adelaide, SA, Australia). All animal experiments were approved by the Women's and Children's Health Research Institute's Animal Ethics Committee and principles of laboratory animal care were followed. Female Flii $^{+/-}$, WT and Flii ${ }^{\text {Tg/Tg }}$ mice of 12-16 weeks of age, weighing 20-35 g were given one intraperitoneal [22] injection of streptozotocin (STZ) (SigmaAldrich, St Louis, MO, USA) at $50 \mathrm{mg} / \mathrm{kg}$ for five consecutive days as described previously [23]. Diabetes was confirmed by assessing the fasting blood glucose levels on 0, 15, 30 and 
60 days post STZ injection. After 6 weeks of confirmed diabetes, excisional wounds were created on the dorsal surface of the mice as described below. Age-matched non-diabetic control animals were treated with an equivalent dose of vehicle.

Murine model of diabetic wound healing Two $6 \mathrm{~mm}$ wounds, one on each side of the midline, were created on the shaved dorsa of mice. Digital photographs were taken of the wounds at $0,7,14$ and 21 days post wounding. Wounds were harvested at $0,7,14$ and 21 days and fixed in $10 \% \mathrm{wt} / \mathrm{vol}$. buffered formalin. In a separate cohort, wounded diabetic WT mice were $(n=10 ; 20$ wounds in total) were injected intradermally around the wound margins with $200 \mu \mathrm{l}$ of FnAb $50 \mu \mathrm{g} / \mathrm{ml}$ at day 1, 2 and 3. Control diabetic mice were treated with an equivalent dosage of $\operatorname{IgG}$ antibodies $(n=10,20$ wounds in total).

Skin explant outgrowth assay Punch biopsies, $2 \mathrm{~mm}$, were taken from the excised skin of diabetic and non-diabetic mice. Biopsies were placed in ice-cold Nutrient Mixture F12 (Sigma-Aldrich) and 1\% (vol./vol.) penicillin-streptomycin solution (Sigma-Aldrich) and cultured as described previously [24] in either normal ( $5.5 \mathrm{mmol} / \mathrm{l})$ or high $(25 \mathrm{mmol} / \mathrm{l})$ glucose media.

Immunohistochemistry Sections were deparaffinised in two changes of xylene and re-hydrated in two changes of absolute alcohol, 1 min each, after which they were transferred into $70 \%$ (vol./vol.) and 30\% (vol./vol.) alcohol for $1 \mathrm{~min}$ each. Sections were brought to water, rinsed in $\times 1$ PBS and placed into $250 \mathrm{ml}$ target retrieval solution as described previously [19]. Primary antibodies (1:100) were applied and incubated overnight at $4{ }^{\circ} \mathrm{C}$. Detection was by species-specific secondary antibodies (1:200). AnalySIS software package (Munster, Germany) was used to measure fluorescence intensity per unit area as described previously [19]. Control immunostaining was carried out by omitting primary or secondary antibodies for verification of staining and non-specific binding.

Aortic ring assay Dissection of the aorta, serum starvation, embedding and feeding of the aortic rings, imaging and quantifying microvessel sprouts was as described previously [25]. A total of 15 rings per mouse were obtained. The microvessel growth from the aorta rings was quantified on days 5, 6, 7 and 8 post embedding by live phase-contrast microscopy. Emerging microvessels were counted and the data was plotted as mean microvessel numbers per ring as described previously [25].

Isolation and culture of HUVEC The collection of primary HUVEC for use in this study was given ethical clearance from relevant committees and consent was obtained from all participants in accordance with the Declaration of Helsinki. HUVEC were isolated from umbilical veins by collagenase digestion as previously described [26] and used at passage two for experiments.

Matrigel tube formation assay Passage 1-2 HUVEC were cultured on gelatine-plated T25 flasks and maintained in culture media containing 10\% (vol./vol.) FCS in EGM-2 BulletKit (Lonza, Basel, Switzerland). HUVEC were seeded on angiogenesis plate (1 $\mu$-Slide Angiogenesis ibiTreat; Ibidi, Munich, Germany) coated with $12 \mu 1$ of Matrigel (354234, BD Biosciences, Bedford, MA, USA). The number of tubes was counted as previously described $[22,27]$ and data were derived from an average of four donors.

Matrigel plug assay Two plugs containing different ligands (FnAb or IgG antibodies were mixed by pipetting; $500 \mu \mathrm{l}$ at $50 \mu \mathrm{g} / \mathrm{ml}$ ) were injected into each mouse to avoid differences between the individual mice [28]. The plug on the left side of the abdomen contained Matrigel compound (354248, BD Biosciences) mixed with $50 \mu \mathrm{g} / \mathrm{ml}$ of FnAb and the plug on the right (control) contained an equivalent dose of rabbit IgG. The Matrigel plugs were removed after 7 days; half of the plug was snap frozen in liquid nitrogen and used for blood vessel visualisation by immunofluorescence and the other half fixed in 10\% (vol./vol.) formalin and processed for histology. Quantification in Matrigel plug assay was as described previously [28] using Image ProPlus 5.1 (Media Cybernetics, Rockville, MD, USA). Matrigel plugs retrieved from $\mathrm{Flii}^{+/-}$, WT and Flii $^{T g / T g}$ mice were fixed in $4 \%$ (wt/vol.) paraformaldehyde/ $1.25 \%(\mathrm{wt} / \mathrm{vol}$.) glutaraldehyde and processed for electron microscopy.

Electron microscopy After washing, specimens were dehydrated in a graded series of ethanol (70-100\%) followed by further dehydration in propylene oxide for $30 \mathrm{~min}$ and treated further as previously described [24]. Electron micrographs of six samples of Matrigel plugs harvested from $\mathrm{Flii}^{+/-}$, WT and Flii ${ }^{T g / T g}$ mice were captured at two different magnifications $(\times 3,800$ and $\times 88,000)$. Blood capillaries were examined focusing on the tight junction region, which seals the intercellular space between adjacent endothelial cells. The overall width of the outer leaflets of the cell membrane of adjacent endothelial cells forming tight junctions was measured using Analysis5 iTEM software (SIS, Tokyo, Japan).

Western blotting Protein was extracted from day 7 WT diabetic mouse wounds treated with either IgG control antibodies or FnAb using standard protein extraction protocols [29]. Protein, $50 \mu \mathrm{g}$, was subjected to SDS-PAGE separation and immunoblotting. Densitometry was performed on bands within the linear range and fold changes in levels calculated from this data as previously described [10]. 
Statistical analysis Statistical differences were determined using the Student's $t$ test to compare between two groups or an ordinary two-way ANOVA with Tukey's multiple comparisons test when comparisons between more than two groups were required. A $p$ value $<0.05$ was considered significant.

\section{Results}

Cytoskeletal protein FLII is increased in human diabetic wounds Wound biopsies were collected from patients diagnosed with diabetic foot ulcers with duration $\geq 6$ weeks. All wounds studied showed features characteristic of diabetic ulcers with a loss of epithelium that extended into the dermis and deeper layers of skin. The epithelial margin was characterised by marked hyperkeratosis. Acute wounds ( $\leq 6$ weeks old) were collected from six patients with acute trauma wounds such as those seen in the emergency room. Unwounded skin specimens were collected from two diabetic patients and six healthy donors and used as controls. Representative haematoxylin and eosin (H\&E)stained views of a diabetic ulcer, acute wound, diabetic unwounded skin and normal skin are shown in Fig. 1a. To assess the role of FLII in diabetic wounds we first stained human acute, diabetic, diabetic unwounded and normal skin for FLII protein. FLII was observed to be present in low levels in the epidermis and dermis of unwounded skin in both healthy donor and diabetic patient skin controls (Fig. 1a). An increase in FLII level was seen in the epidermis of acute wounds when compared with unwounded skin ( $p \leq 0.01$; Fig. 1b). However, a much greater and more statistically significant increase in FLII was observed in diabetic wounds, especially in the dermis, when compared with acute non-diabetic wounds ( $p \leq 0.001$; Fig. 1b). Considering FLII levels are low in unwounded diabetic patient skin and unwounded skin of healthy donors (Fig. 1a, b), this suggests a specific increase in FLII in response to the development of chronic wounds rather than the onset of diabetes.

Inhibitory effect of FLII on cellular growth and migration Skin explants $(2 \mathrm{~mm})$ were collected from diabetic and non-diabetic mice and incubated in normal $(5.5 \mathrm{mmol} / \mathrm{l})$ or high $(25 \mathrm{mmol} / \mathrm{l})$ glucose-supplemented media for 7 days (Fig. $1 \mathrm{~d}-\mathrm{h}$ ). On day 2 post culture a halo appeared around the explants. The cellular growth and migration potential was determined by measuring the distance between the explant and the end of the growing halo. Diabetic explants displayed a significant reduction in their capacity to grow and migrate compared with non-diabetic skin tissue (Fig. 1e, f).

FLII deficiency improves diabetic wound healing Mice expressing low $\left(\right.$ Flii $\left.^{+/-}\right)$, normal $\left(\right.$Flii $\left.^{+/+}\right)$and high $\left(\right.$Flii $\left.^{T g / T g}\right)$ levels of FLII were treated with STZ and the effect on blood glucose levels determined (Fig. 1c). STZ-treated mice had consistently higher fasting blood glucose levels ( $\geq 14.9 \mathrm{mmol} / \mathrm{l})$ than vehicle controls $(\leq 7.5 \mathrm{mmol} / \mathrm{l})$. Changes in Flii gene expression did not affect blood glucose levels in diabetes (Fig. 1c). Healing was significantly impaired in all three diabetic Flii mice lines (Fig. 2a, b, d) compared with non-diabetic controls (electronic supplementary material [ESM] Fig. 1a, b and Fig. 2c). Excisional wounds were created on the backs of low $\left(\right.$ Flii $\left.^{+/-}\right)$, normal $\left(\right.$Flii $\left.^{+/+}\right)$and high $\left(F l i i^{T g / T g}\right)$ FLII mice; representative digital images of 0 , 7, 14 and 21 day non-diabetic and diabetic wounds are shown in ESM Figs 1a and 2a, respectively. Decreased Flii gene expression resulted in a significant reduction in wound size in both non-diabetic and diabetic wounds as determined by the planimetric analysis of surface wound area (ESM Fig. 1a and Fig. 2a, c, d, respectively). Histological analysis of diabetic wounds revealed that the distance between the dermal wound margins was greater in wounds with high levels of FLII at day 7 compared with WT and FLII-deficient wounds (Fig. 2b, f). The percentage of wound re-epithelialisation was evaluated by measuring the length of the neoepidermis at day 7 after wounding (Fig. 2e).

Diabetes increased FLII level in response to wounding The influence of diabetes and the effect of wounding on FLII protein expression were determined in non-diabetic and diabetic $\mathrm{Flii}^{+/-}$, WT and $\mathrm{Flii}^{\mathrm{Tg} / \mathrm{Tg}}$ mice, at 0,7 and 14 days post wounding (Fig. 3a, b). In unwounded skin, diabetes did not change FLII production when compared with non-diabetic conditions (Fig. 3a, b). Diabetes further increased FLII in response to wounding (Fig. 3c). In diabetic mice, wounding upregulated the FLII protein level by $83 \%$ in $\mathrm{Flii}^{+-}$mice, by $86 \%$ in WT and by $89 \%$ in Flii ${ }^{T g / T g}$ mice (Fig. 3c). Significantly less FLII staining was observed in diabetic Flii ${ }^{+/}$ wounds at days 7 and 14 post wounding compared with diabetic Flii ${ }^{T g / T g}$ wounds (Fig. 3c).

FLII deficiency leads to upregulation of pro-angiogenic VEGF expression in diabetic wounds Day 0, 7 and 14 wounds from non-diabetic and diabetic (ESM Fig. 2a, b) $\mathrm{Flii}^{+/}$, WT and Flii ${ }^{T g / T g}$ mice were stained for VEGF expression. Flii overexpression caused a significant reduction in VEGF at both day 7 and day 14 post wounding, with VEGF level in Flii $^{\mathrm{Tg} / \mathrm{Tg}}$ non-diabetic wounds being fivefold lower compared with non-diabetic WT at 7 days post wound (Fig. 4a). FLII deficiency was associated with a significant increase in VEGF expression in day $0 \mathrm{WT}$ diabetic wounds compared with day 0

Flii $^{T g / T g}$ diabetic wounds (ESM Fig. 2b and Fig. 4a). A significant increase in VEGF expression in diabetic Flii ${ }^{+/-}$ wounds compared with diabetic WT at day 7 post wounding was observed (Fig. 4a).

Increased levels of FLII leads to increased numbers of endothelial cells in diabetic wounds To determine the effect of 
Fig. 1 FLII is increased in human diabetic wounds and its deficiency downregulates cellular migration. (a) Representative H\&E and FLII (green) and DAPI (blue) nuclear counterstain immunofluorescence in normal unwounded skin, acute wounds, diabetic unwounded skin and diabetic wounds. All images magnification $\times 10$, scale bars $50 \mu \mathrm{m}$. (b) Graphical representation of the intensity of FLII staining in the four groups. (c) Mice expressing low $\left(\right.$ Flii $\left.^{+/-}\right)$ (white bars), normal (WT) (grey bars) and high $\left(F_{i i i^{T g / T g}}\right)$ (black bars) levels of FLII were treated with five consecutive daily injections of $50 \mathrm{mg} / \mathrm{kg} \mathrm{STZ}$ or sodium citrate buffer. Fasting blood glucose levels were determined by tail-vein sampling and data obtained on $0,15,30$ and 60 days post STZ injection. (d) Representative day 7 micrographs showing cellular migration on in vitro incubation of skin explants from diabetic and nondiabetic Flii mice; $n=4$. Scale bar $100 \mu \mathrm{m}$. (e-h) Graphical representation of $\mathrm{Flii}^{+/-}$(circles), WT (squares) and Flii ${ }^{\text {Tg/Tg }}$ (triangles) cellular outgrowth under non-diabetic $(5 \mathrm{mmol} / 1$ glucose) (e) and diabetic $(25 \mathrm{mmol} / \mathrm{l}$ glucose) (f) conditions as well as osmotic controls (non-diabetic $25 \mathrm{mmol} / 1$ glucose) (g) and (diabetic $5 \mathrm{mmol} / 1$ glucose) (h). All values represent means and SEM; $n=6$. ${ }^{* * *} p \leq 0.01$ and ${ }^{* * *} p \leq 0.001$. D, dermis; E, epidermis; UNW, unwounded a

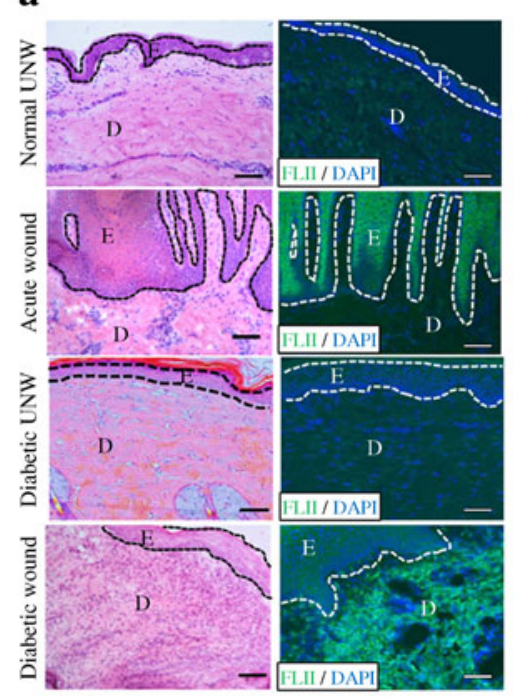

b
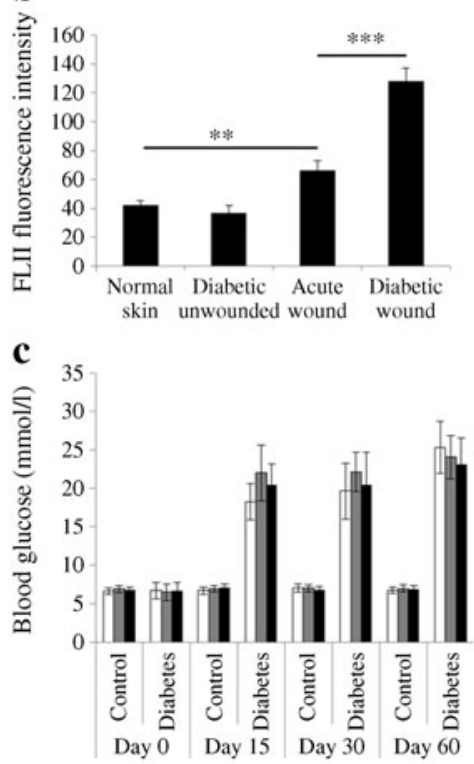

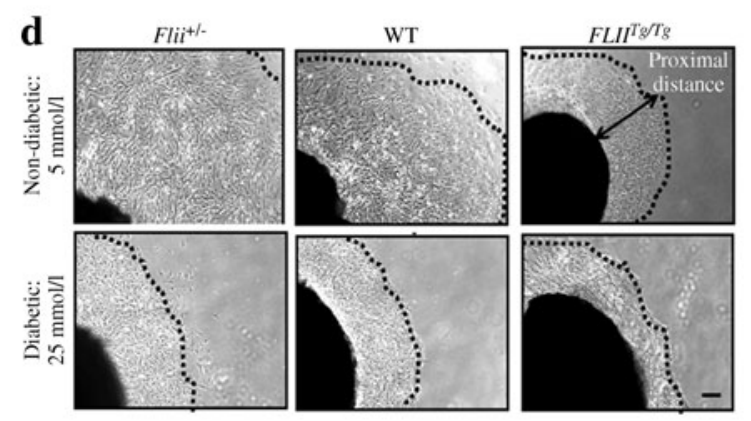

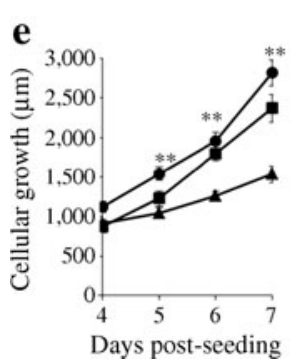

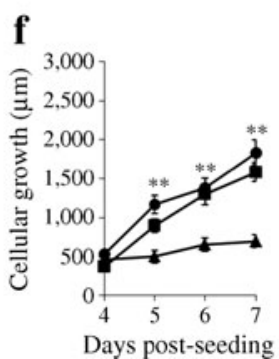

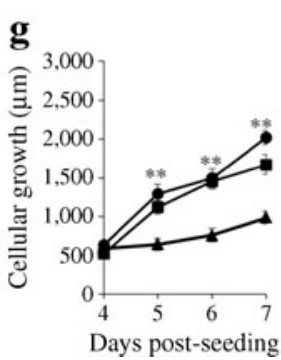

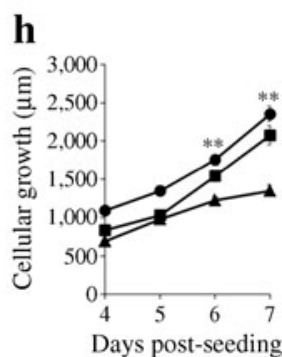

FLII on endothelial cell recruitment, healing wounds from non-diabetic and diabetic $\mathrm{Flii}^{+/-}$, WT and Flii ${ }^{\mathrm{Tg} / \mathrm{Tg}}$ mouse wounds were stained for vWF (ESM Fig. 2c and Fig. 4b) and CD31 (ESM Fig. 2f, g and Fig. 4c, d). Day 7 non-diabetic mice Flii $^{+/}$wounds showed a 1.7-fold increase in the expression of vWF (Fig. 4b) and CD31 (Fig. 4c) compared with WT as determined by immunohistochemistry. Low FLII led to markedly increased endothelial cell numbers in diabetic wounds compared with diabetic WT controls (Fig. 4b, d).

Attenuation of Flii gene expression improves new blood vessel formation in vivo Matrigel plugs were injected under the skin of $\mathrm{Flii}^{+-}$, WT and Flii ${ }^{\mathrm{Tg} / \mathrm{Tg}}$ mice to determine the effect of
FLII on angiogenesis (Fig. 5a-c). The average lengths of newly formed vessels and vessel-like structures that had grown into the Matrigel plugs were quantified (Fig. 5b). The length of newly formed vessels was quantified and categorised into three groups as previously described [28]: (1) vessels containing erythrocytes; (2) vessels without erythrocytes; and (3) vessel-like structure but without a cavity. In this in vivo model of angiogenesis, reduced levels of FLII resulted in a fourfold increase in the length of functional vessels containing erythrocytes compared with WT (Fig. 5b). The length of vessels with cavities, but without the erythrocytes, was significantly higher in $\mathrm{Flii}^{+-}$mice than their WT counterparts (Fig. 5b). Electron microscopy revealed 
Fig. 2 Flii overexpression impairs healing in mouse diabetic wounds. (a) Representative images of full-thickness excisional wounds on the backs of STZ-induced Flii diabetic mice on days $0,7,14$ and 21 post surgery. (b) Representative images of H\&E-stained sections of diabetic wounds at 7 and 21 days after wounding. Arrows indicate the wound margins. All images magnification $\times 4$, scale bars $100 \mu \mathrm{m}$. Graphical representation of surface wound area at $0,7,14$ and 21 days after wounding in $\mathrm{Flii}^{+/-}$(white bars), WT (grey bars) and Flii ${ }^{T g / T g}$ (black bars) non-diabetic control mice (c) and STZ-induced diabetic mice (d). Graphical representation of wound reepithelialisation (e) and dermal gape (f) in $\mathrm{Flii}^{+-}$(white bars), WT (grey bars) and Flii ${ }^{T g / T g}$ (black bars) non-diabetic and diabetic mice at day 7 after surgery. All values represent means and SEM; $n=6$ mice, 12 wounds each group/ timepoint. $p \leq 0.05$ and ${ }^{* *} p \leq 0.01$
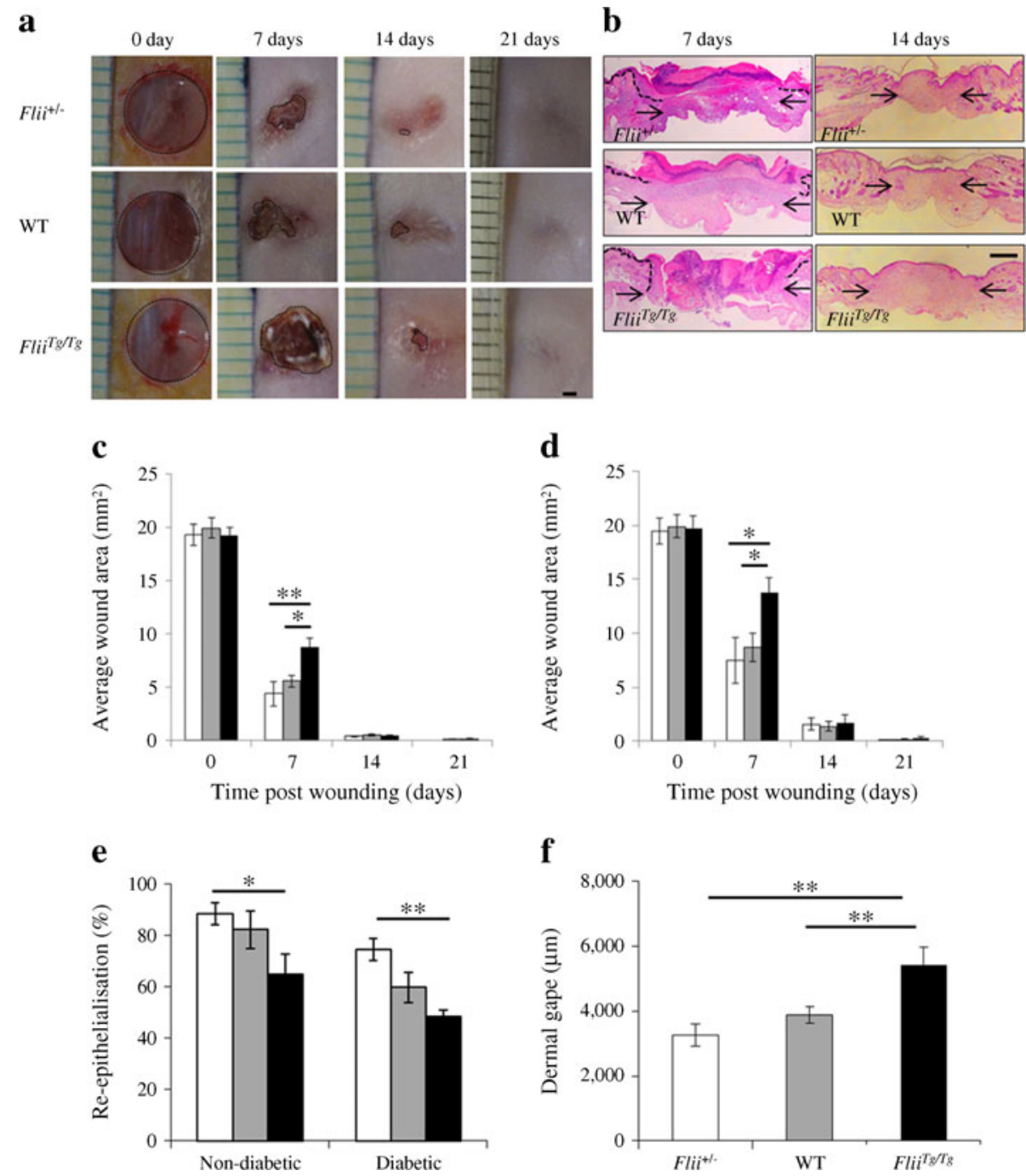

that the endothelial cells in the Flii ${ }^{T g / T g}$ group showed disrupted tight junction formation (Fig. 5a). The distances between the nearest edges of endothelial cells and their tight junctions were measured (Fig. 5c). Partial knockout of the Flii gene had a positive effect on tight junction formation and resulted in significantly smaller distances between adjacent endothelial cells compared with the Flii ${ }^{T g / T g}$ group (Fig. 5c). Aortas collected from 4-week-old $\mathrm{Flii}^{+/-}$, WT and Flii ${ }^{\mathrm{Tg} / \mathrm{Tg}}$ mice were cut into rings and embedded in type 1 collagen (Fig. 5d). Endothelial microvessel sprouts grown from the rings were counted at days $0,5,6,7$ and 8 following embedding. Significant increases in microvessel sprouting were observed in $\mathrm{Flii}^{+/-}$rings compared with WT controls at day 5 and day 6 post embedding (Fig. 5e). Significantly reduced sprouting was observed in Flii ${ }^{T g / T g}$ rings isolated from the aortas of $\mathrm{Flii}^{+/-}$and WT mice (Fig. 5e).

Pro-angiogenic effects of FnAb in vitro To determine whether $\mathrm{FnAb}$ had the capacity to enhance capillary tube formation in vitro, we plated HUVEC on a two-dimensional matrix of
Matrigel in the presence of FnAb $(50 \mu \mathrm{g} / \mathrm{ml})$ or an isotypematched control (irrelevant $\mathrm{IgG}$ ) at a final concentration of $50 \mu \mathrm{g} / \mathrm{ml}$ (ESM Fig. 3a, b). Assessment of capillary tube formation of the FnAb-treated HUVEC showed that these cells reorganised into large aggregates with subsequent capillary tubes (ESM Fig. 3a, b). The FnAb-treated HUVEC formed significantly more capillary tubes compared with IgG-treated controls or no treatment (Fig. 6a).

\section{Reducing FLII using FnAb enhanced formation of mature} blood vessels in vivo Matrigel plugs were mixed with either IgG control $(50 \mu \mathrm{g} / \mathrm{ml})$ or $\mathrm{FnAb}(50 \mu \mathrm{g} / \mathrm{ml})$ and injected under the skin of the abdominal area of WT mice (ESM Fig. 3c, d and Fig. 6b). The vessels were categorised into three groups: (1) vessels containing erythrocytes; (2) vessels without erythrocytes; and (3) vessel-like structure but without a cavity. $\mathrm{FnAb}$ resulted in a fourfold increase in the length of functional vessels that contained erythrocytes compared with IgG control (Fig. 6b). Adding FnAb (50 $\mu \mathrm{g} / \mathrm{ml})$ into Matrigel plugs enhanced the formation of new blood vessels (Fig. 6b). 


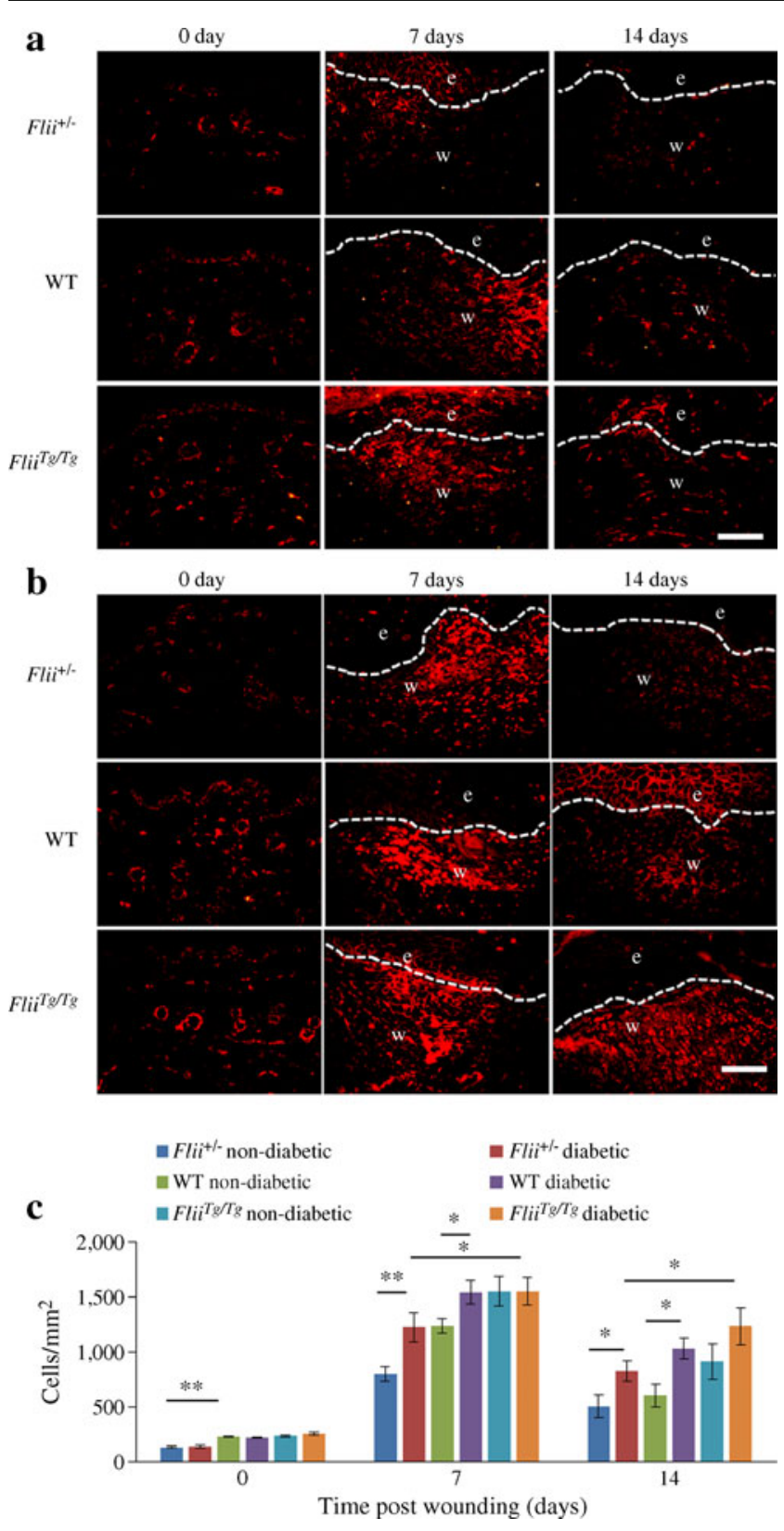

Fig. 3 FLII is upregulated in response to wounding. Representative images of FLII immunofluorescence in $\mathrm{Flii}^{+/-}$, WT and Flii ${ }^{\mathrm{Tg} / \mathrm{Tg}}$ nondiabetic (a) and diabetic (b) mice excisional wounds at day 0, 7 and 14 following surgery. All images magnification $\times 20$. Scale bars in (a) and (b), $50 \mu \mathrm{m}$, refer to all images. (c) Graphical representation of FLII fluorescence intensity in epidermis and dermis of non-diabetic and diabetic mice wounds at day 0,7 and 14 following surgery. All values represent means and SEM; $n=6$ mice, 12 wounds each group/timepoint. ${ }^{*} p \leq 0.05$ and ${ }^{* *} p \leq 0.01$

FnAb contributes to enhanced $\alpha-S M A^{+} / C D 31^{+}$cell recruitment in vivo Matrigel plugs supplemented with either IgG control $(50 \mu \mathrm{g} / \mathrm{ml})$ or FnAb $(50 \mu \mathrm{g} / \mathrm{ml})$ were injected under the skin of WT mice and harvested at day 7 post injection and subsequently stained with antibodies to establish the level of $\alpha$-SMA and endothelial cell $\left(\mathrm{CD} 31^{+}\right)$recruitment (Fig. 6c). The number of cells positive for $\alpha$-SMA was lower in IgG- treated Matrigel plugs compared with FnAb-treated Matrigel plug sections (Fig. 6d).

FLII antibodies increase VEGF expression in diabetic wounds The effect of reduced FLII on VEGF levels in diabetic wounds was assessed by immunoblotting and immunostaining with anti-VEGF antibody the day 7 WT diabetic wounds treated with either IgG control antibodies or FnAb (ESM Fig. 3e and Fig. 6e, f). FnAb resulted in significant upregulation of VEGF (Fig. 6e-g).

Accelerated diabetic wound closure is induced by FnAb in vivo FnAb were injected intradermally into WT mice and marked differences were recorded in the macroscopic appearance of healing wounds at $0,1,2,3$ and 7 days after wounding (ESM Fig. 4a and Fig. 7a-d). By the end of the first week the control wounds were still covered with a non-viable necrotic tissue which included the clot (ESM Fig. 4a). In contrast, the appearance of wounds treated with FnAb was markedly improved compared with control wounds, i.e. faster clearance of non-viable tissue and rapid appearance of granulation tissue, which was promptly replaced by shiny epidermal layer (ESM Fig. 4a). Intradermal administration of $\mathrm{FnAb}$ $(200 \mu \mathrm{l}$ of $50 \mu \mathrm{g} / \mathrm{ml}$ injected at day 1,2 and 3 post wounding), but not control antibodies (irrelevant affinity purified $\mathrm{IgG} ; 200 \mu \mathrm{l}$ of $50 \mu \mathrm{g} / \mathrm{ml}$ injected at day 1,2 and 3 post wounding), resulted in a 1.9-fold decrease in wound healing (Fig. 7b). Microscopic analysis of wound biopsies showed that intradermal injection of $\mathrm{FnAb}$ accelerated wound closure compared with IgG-treated counterparts. FnAb-treated wounds had significantly smaller wound length and gape than IgG-treated control wounds on day 7 after wounding (Fig. 7c, d).

\section{Discussion}

Human diabetic wounds have elevated levels of FLII, a cytoskeletal protein previously shown to inhibit cellular migration, adhesion and proliferation and to negatively impact on the wound repair process [17]. To determine the extent of the involvement of FLII in diabetic wound healing, mice with low $\mathrm{Flii}^{+/-}$, normal (WT) and high (Flii ${ }^{T g / T g}$ ) expression of Flii gene were rendered diabetic and assessed for their healing characteristics. Diabetes was associated with a delayed wound healing phenotype with larger wound area and, as observed in human patients, elevated levels of FLII in the wounds. Reduced FLII significantly improved the healing of diabetic and non-diabetic wounds, whereas FLII overexpression decreased the rate of re-epithelialisation under both diabetic and nondiabetic conditions. Given that rapid epithelialisation and closure of disrupted dermal matrix are key determinants of 


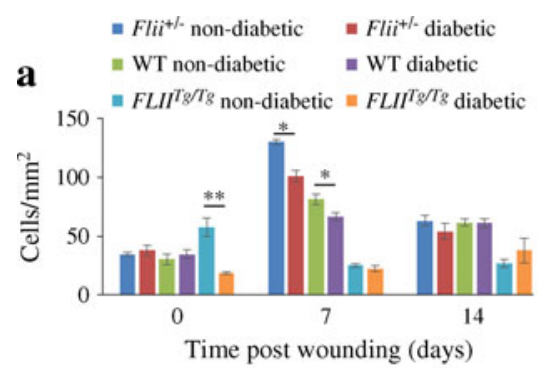

c

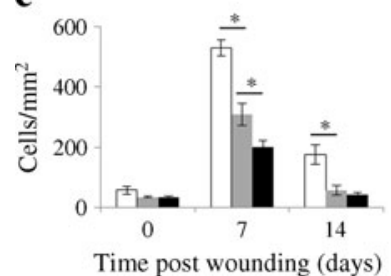

Fig. 4 Effects of FLII on VEGF, vWF and CD31 expression in nondiabetic and diabetic wounds. Graphical representation of VEGF (a) and vWF (b) immunofluorescence-positive cells $/ \mathrm{mm}^{2}$ in excisional wounds in $\mathrm{Flil}^{++}$, WT and Flii ${ }^{\mathrm{Tg} / \mathrm{Tg}}$ non-diabetic and diabetic mice at days 0,7 and 14 after surgery. Graphical representation of CD31-

successful wound healing these results suggest that decreasing the level of FLII in diabetic wounds may exert a positive effect on wound healing.

Diabetic wounds fail to heal because of several contributing factors, including abnormal keratinocyte and fibroblast migration, proliferation, differentiation, and apoptosis and decreased vascularisation [3]. Given that FLII inhibits cellular migration [3] and vascular cell migration is an important component of new vessel formation and vascular maturation [30] we investigated whether altering Flii gene expression could affect angiogenesis in diabetic wounds. Diabetes itself caused a downregulation in both VEGF and vWF during the early stages of wound healing, which in turn coincided with high levels of FLII. New blood vessel formation was further impaired in FLII-overexpressing diabetic wounds, with reduced numbers of endothelial cells and decreased VEGF and vWF being observed. In FLII-deficient diabetic wounds, the levels of vWF were elevated twofold at days 7 and 14, suggesting that lowering the level of FLII in diabetic wounds could have a positive effect on angiogenesis and therefore healing. In support of this, robust sprouting of endothelial cells was observed from aortic rings obtained from $\mathrm{Flii}^{+/-}$ mice, whereas in contrast aortic rings from $\mathrm{Flii}^{\mathrm{Tg} / \mathrm{Tg}}$ mice showed minimal microvessel outgrowth suggesting that FLII inhibits angiogenic processes. FLII deficiency was also associated with enhanced cell-to-cell interactions between endothelial cells with physiologically normal tight junctions being observed when FLII levels were reduced, and disrupted cellto-cell interactions with significantly wider distances observed in FLII-overexpressing mice wounds. These findings support previous observations of impaired hemidesmosome formation

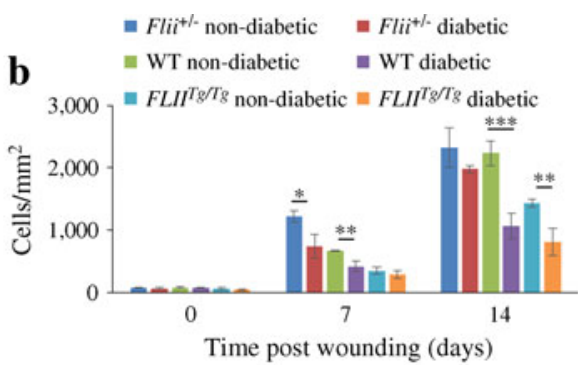

d

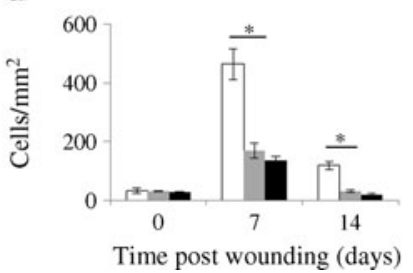

immunofluorescence-positive cells $/ \mathrm{mm}^{2}$ in non-diabetic (c) and diabetic (d) wounds of Flii ${ }^{+/-}$(white bars), WT (grey bars) and Flii ${ }^{T g / T g}$ (black bars) mice at day 0,7 and 14 post surgery. All values represent means and SEM; $n=6$ mice, 12 wounds each group/timepoint. ${ }^{*} p \leq 0.05$ and ${ }^{* *} p \leq 0.01$

in FLII-overexpressing mice [24] and suggest the importance of FLII in endothelial cell tight junction formation, which is important for blood vessel stability and leakage prevention.

FLII is secreted by both fibroblasts and macrophages [10, 17] and is present in acute and chronic human wound fluid [18]. FLII is secreted through a non-classic late endosome/ lysosome-mediated pathway by at least two of the major cell types found in wounds, fibroblasts and macrophages [10], and is able to bind to lipopolysaccharide and alter macrophage activation [10]. Treatment of wound fluid with FnAb decreases the level of available FLII present in the wound fluid, which implies that FnAb can 'mop up' extracellular FLII and neutralise its activity in vivo [20].

As wounds in FLII-deficient mice showed improved healing, we assessed the effect of using FnAb as a means of decreasing local levels of FLII within the wound environment. Diabetic wounds treated with FnAb showed accelerated wound closure with a 1.9-fold decrease in average wound size observed in day 3 and day 7 WT FnAb-treated diabetic wounds. Similar effects have been reported in previous studies where FnAb improved the rate of incisional [17], excisional [20] and burn wound repair [19]. Dose-dependent effects of $\mathrm{FnAb}$ on cellular proliferation have also previously been demonstrated [20] and the addition of FnAb to human chronic wound fluids can reduce their inhibitory effect on cell proliferation in vitro [18]. Both of these effects could contribute positively to improved wound outcomes.

Angiogenic responses to the FnAb were also assessed, with $\mathrm{FnAb}$ leading to significantly increased capillary tube formation and longer and more mature blood vessel formation compared with IgG controls. FnAb-treated Matrigel plugs 

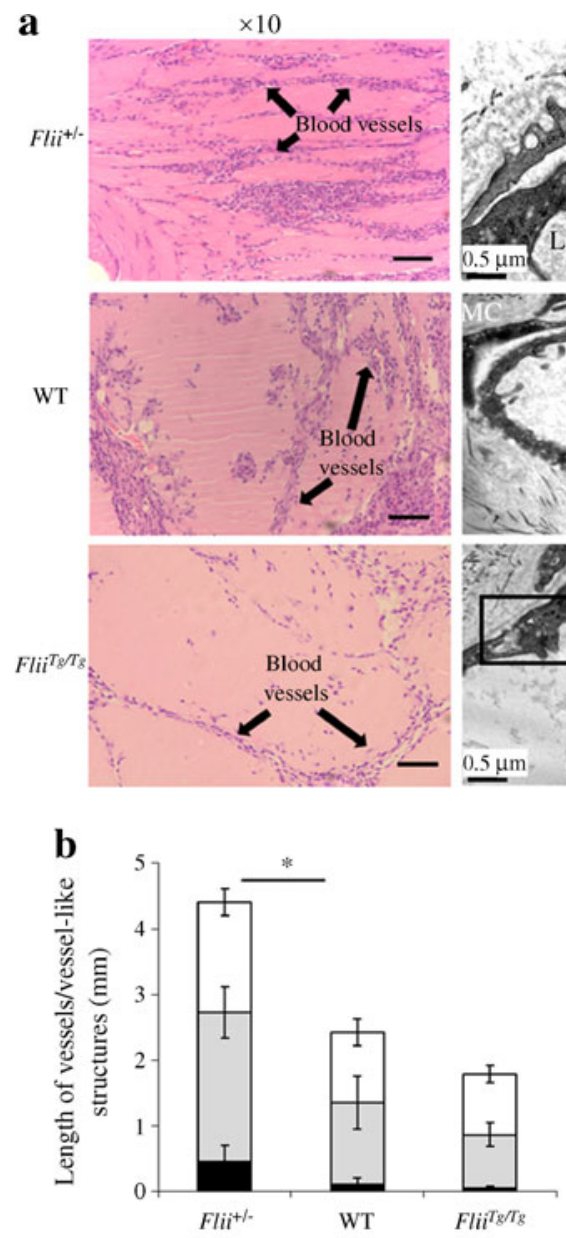

d

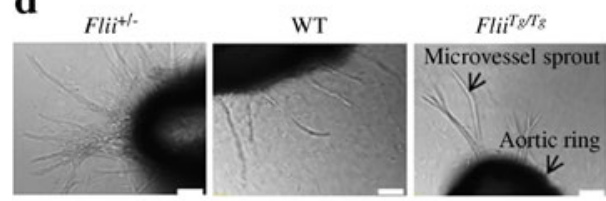

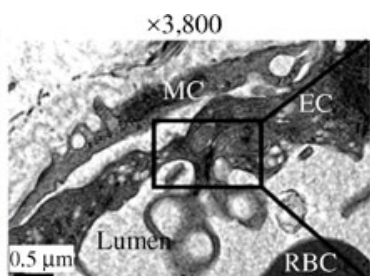
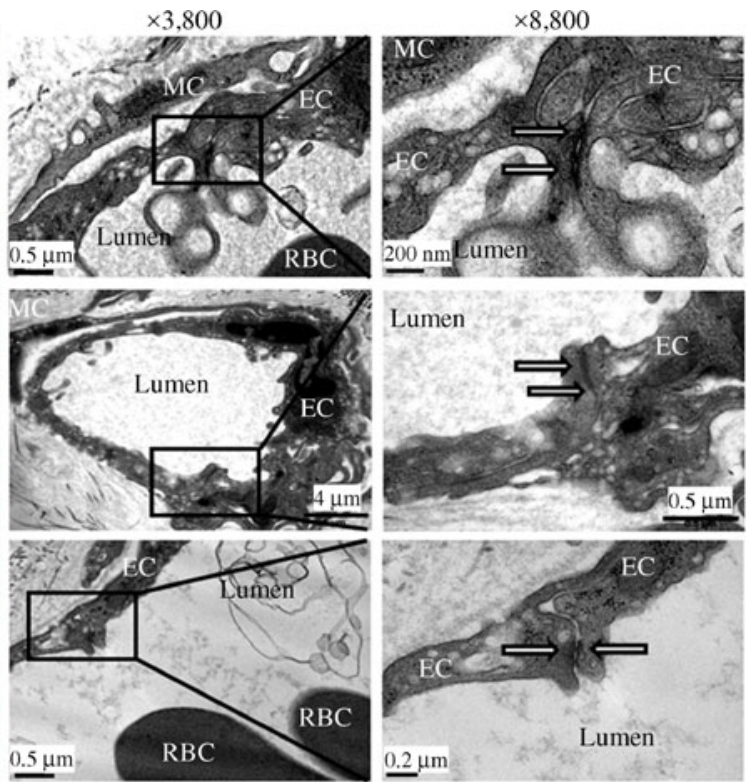

c
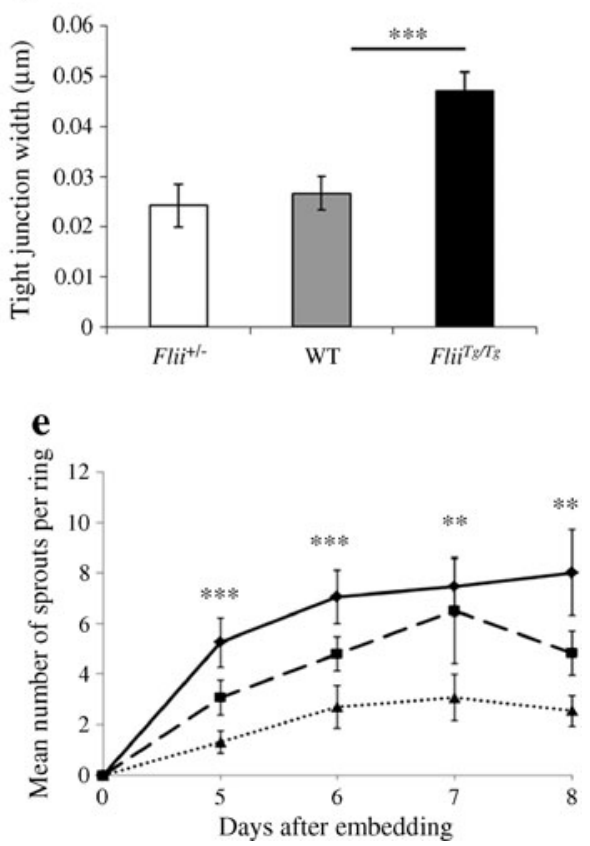

and (2) vessels without cavities (white bars). (c) Quantification of the distance between adjacent endothelial cells. $n=6 .{ }^{* * *} p \leq 0.001$. (d) Phasecontrast images of aortic rings embedded in type 1 collagen showing microvessel outgrowth at day 6 after embedding. Scale bars $100 \mu \mathrm{m}$. Magnification $\times 10$. (e) Time course of microvessel sprouting from aortic rings collected from aortas of $\mathrm{Flii}^{+/}$(solid line), WT (dashed line) and Flii $^{T g / T g}$ (dotted line) mice aged 4 weeks. Results represent the mean number of microvessel sprouts and SEM. Assay quantification $(n=6 ; 15$ rings per mouse aorta). ${ }^{* *} p \leq 0.01 ; \mathrm{Flii}^{+/-}$and WT vs Flii ${ }^{\mathrm{Tg} / T g}$ on days $5-$ 8. ${ }^{* * *} p \leq 0.001, F_{i i}{ }^{+/-}$vs WT on days 5 and 6 . MC, mural cells; EC, endothelial cells; RBC, erythrocytes

flow, with flow being a critical determinant of vessel maintenance and durability [31]. In the Matrigels treated with FnAb, 


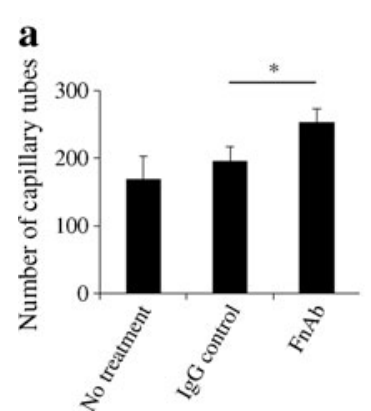

b

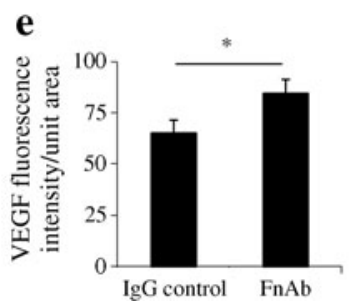

Fig. 6 New vessel formation is enhanced when FLII levels are reduced. (a) Graphical representation of capillary tube numbers $6 \mathrm{~h}$ following no treatment, dose-matched IgG control or FnAb treatment of HUVEC grown on Matrigel substrate in vitro. (b) Quantification of the formation of new vessels in Matrigel plugs in vivo by analysis of vessel lengths. Representative images of platelet/endothelial cell adhesion molecule 1 (PECAM1) (CD31)- and $\alpha$-SMA-immunofluorescence-positive vessels,

the expression of $\alpha$-SMA-positive cells was also significantly higher, suggesting that $\mathrm{FnAb}$ may be able to promote the

a
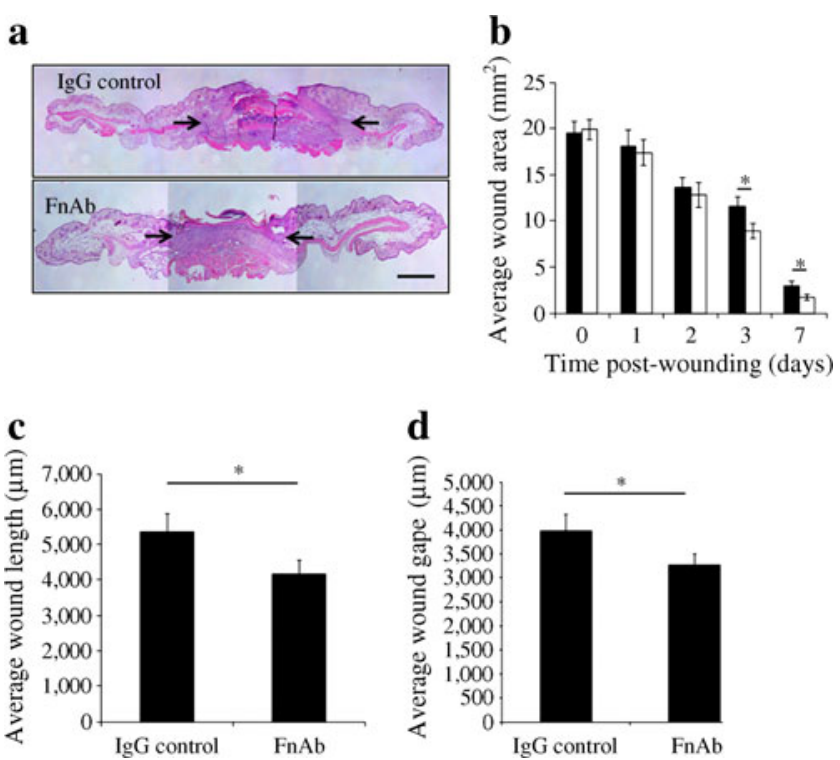

Fig. 7 Positive effect of FnAb treatment on diabetic wound healing. (a) Representative images of H\&E-stained IgG control or FnAb-treated diabetic WT wounds at day 7 post surgery. Scale bar $1 \mathrm{~mm}$. Black arrows point at wound margins. Graphical analysis of microscopic measurements of histological wound variables including average wound area (b) (black bars, IgG-control-treated wounds; white bars, FnAb-treated wounds); average wound length (c) and wound gape (d) following IgG control or $\mathrm{FnAb}$ treatment of WT wounds. $n=6$; two wounds/mouse; 12 wounds. All values represent means and SEM. ${ }^{*} p \leq 0.05$

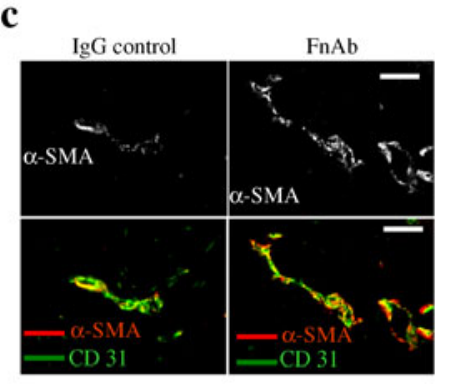

d

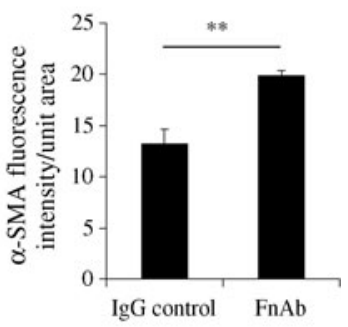

green and red, respectively (c); and graphical representation of $\alpha$-SMA (d) fluorescence intensity in vessels grown in Matrigel plugs containing either control IgG or FnAb treatment. $n=6$. Graphical representation (e), western blotting analysis (f) and band densitometry (g) of VEGF expression in day 7 diabetic WT wounds treated with control IgG or FnAb. Figure represents data from three replicate experiments. $n=4$. All values represent means and SEM. ${ }^{*} p \leq 0.05$ and ${ }^{* *} p \leq 0.01$

migration of these cells towards endothelial cells, hence stimulating the formation of functionally mature blood vessels.

In summary, the actin cytoskeletal protein FLII is upregulated in both human and mouse diabetic wounds. Reducing its activity either by genetic knockdown or by the application of neutralising antibodies leads to improved wound healing. One mechanism via which FLII may be actively promoting improved wound healing is via its pro-angiogenic effects. Identification of novel wound-healing targets and understanding how these targets may affect wound healing is the first step in the development of new therapies that will improve the quality of life of patients with diabetes and reduce the costs of diabetic wound management.

Acknowledgements The authors thank L. Waterhouse for excellent electron microscopy expertise, the Animal Care Facility at the Women's and Children's Hospital, Adelaide, the mothers and staff at the Women's \& Children's Hospital, and Burnside War Memorial Hospital for collection of umbilical cords.

Funding AJC is supported by the National Health and Medical Research Council Senior Research Fellowship (number 1002009). ZK is supported by the National Health and Medical Research Council Early Career Fellowship (number 1036509). CSB is supported by a Heart Foundation Fellowship.

Duality of interest AJC is a shareholder in a company developing intellectual property associated with FnAb treatment of wounds. All other authors declare that there is no duality of interest associated with their contribution to this manuscript. 
Contribution statement NR and ZK made substantial contribution to the design, acquisition and analysis of data and have drafted the manuscript. EM, SLA, CSB, RMA and RF made substantial contribution to the interpretation of data and revision of the manuscript. AJC made substantial contribution to the design and drafting of the manuscript. All authors have reviewed and approved the final version.

\section{References}

1. Brem H, Tomic-Canic M (2007) Cellular and molecular basis of wound healing in diabetes. J Clin Invest 117:1219-1222

2. Oyibo SO, Jude EB, Tarawneh I, Nguyen HC, Harkless LB, Boulton AJ (2001) A comparison of two diabetic foot ulcer classification systems: the Wagner and the University of Texas wound classification systems. Diabetes Care 24:84-88

3. Albiero M, Menegazzo L, Boscaro E, Agostini C, Avogaro A, Fadini GP (2011) Defective recruitment, survival and proliferation of bone marrow-derived progenitor cells at sites of delayed diabetic wound healing in mice. Diabetologia 54:945-953

4. Xue W, Cai L, Tan Y et al (2010) Cardiac-specific overexpression of HIF-1 $\alpha$ prevents deterioration of glycolytic pathway and cardiac remodeling in streptozotocin-induced diabetic mice. Am J Pathol 177:97-105

5. Carmeliet P, Jain RK (2011) Molecular mechanisms and clinical applications of angiogenesis. Nature 473:298-307

6. Davy DA, Ball EE, Matthaei KI, Campbell HD, Crouch MF (2000) The flightless I protein localizes to actin-based structures during embryonic development. Immunol Cell Biol 78:423-429

7. Davy DA, Campbell HD, Fountain S, de Jong D, Crouch MF (2001) The flightless I protein colocalizes with actin- and microtubule-based structures in motile Swiss 3T3 fibroblasts: evidence for the involvement of PI 3-kinase and Ras-related small GTPases. J Cell Sci 114: $549-562$

8. Goshima M, Kariya K, Yamawaki-Kataoka Y et al (1999) Characterization of a novel Ras-binding protein Ce-FLI-1 comprising leucine-rich repeats and gelsolin-like domains. Biochem Biophys Res Commun 257:111-116

9. Campbell HD, Fountain S, McLennan IS et al (2002) Fliih, a gelsolin-related cytoskeletal regulator essential for early mammalian embryonic development. Mol Cell Biol 22:3518-3526

10. Lei N, Franken L, Ruzehaji N, Offenhauser C, Cowin AJ, Murray RZ (2012) Flightless, secreted through a late endosome/lysosome pathway, binds LPS and dampens cytokine secretion. J Cell Sci 125:4288-4296

11. Bella J, Hindle KL, McEwan PA, Lovell SC (2008) The leucine-rich repeat structure. Cell Mol Life Sci 65:2307-2333

12. Kobe B, Kajava AV (2001) The leucine-rich repeat as a protein recognition motif. Curr Opin Struct Biol 11:725-732

13. Wang T, Chuang TH, Ronni T et al (2006) Flightless I homolog negatively modulates the TLR pathway. J Immunol 176:1355-1362

14. Dai P, Jeong SY, Yu Y et al (2009) Modulation of TLR signaling by multiple MyD88-interacting partners including leucine-rich repeat Fli-I-interacting proteins. J Immunol 182:3450-3460
15. Hayashi T, Funato Y, Terabayashi T et al (2010) Nucleoredoxin negatively regulates Toll-like receptor 4 signaling via recruitment of flightless-I to myeloid differentiation primary response gene (88). J Biol Chem 285:18586-18593

16. Li J, Yin HL, Yuan J (2008) Flightless-I regulates proinflammatory caspases by selectively modulating intracellular localization and caspase activity. J Cell Biol 181:321-333

17. Cowin AJ, Adams DH, Strudwick XL et al (2007) Flightless I deficiency enhances wound repair by increasing cell migration and proliferation. J Pathol 211:572-581

18. Ruzehaji N, Grose R, Krumbiegel D et al (2012) Cytoskeletal protein Flightless (Flii) is elevated in chronic and acute human wounds and wound fluid: neutralizing its activity in chronic but not acute wound fluid improves cellular proliferation. Eur J Dermatol 22:740-750

19. Adams DH, Ruzehaji N, Strudwick XL et al (2009) Attenuation of flightless I, an actin-remodelling protein, improves burn injury repair via modulation of transforming growth factor (TGF)-betal and TGFbeta3. Br J Dermatol 161:326-336

20. Jackson JE, Kopecki Z, Adams DH, Cowin AJ (2012) Flii neutralizing antibodies improve wound healing in porcine preclinical studies. Wound Repair Regen 20:523-536

21. Thomsen N, Chappell A, Ali RG et al (2011) Mouse strains for the ubiquitous or conditional overexpression of the Flii gene. Genesis 49: 681-688

22. Barrett JM, Parham KA, Pippal JB et al (2011) Over-expression of sphingosine kinase-1 enhances a progenitor phenotype in human endothelial cells. Microcirculation 18:583-597

23. Johnson MS, Ryals JM, Wright DE (2008) Early loss of peptidergic intraepidermal nerve fibers in an STZ-induced mouse model of insensate diabetic neuropathy. Pain 140:35-47

24. Kopecki Z, Arkell R, Powell BC, Cowin AJ (2009) Flightless I regulates hemidesmosome formation and integrin-mediated cellular adhesion and migration during wound repair. J Invest Dermatol 129: 2031-2045

25. Baker M, Robinson SD, Lechertier T et al (2012) Use of the mouse aortic ring assay to study angiogenesis. Nat Protoc 7:89-104

26. Litwin M, Clark K, Noack L et al (1997) Novel cytokine-independent induction of endothelial adhesion molecules regulated by platelet/ endothelial cell adhesion molecule (CD31). J Cell Biol 139:219-228

27. Bonder CS, Sun WY, Matthews T et al (2009) Sphingosine kinase regulates the rate of endothelial progenitor cell differentiation. Blood 113:2108-2117

28. Kano MR, Morishita Y, Iwata C et al (2005) VEGF-A and FGF-2 synergistically promote neoangiogenesis through enhancement of endogenous PDGF-B-PDGFRbeta signaling. J Cell Sci 118:37593768

29. Kopecki Z, O’Neill GM, Arkell RM, Cowin AJ (2011) Regulation of focal adhesions by flightless $i$ involves inhibition of paxillin phosphorylation via a Rac1-dependent pathway. J Invest Dermatol 131: $1450-1459$

30. Francis-Goforth KN, Harken AH, Saba JD (2010) Normalization of diabetic wound healing. Surgery 147:446-449

31. Carmeliet P (2003) Angiogenesis in health and disease. Nat Med 9: 653-660 